\title{
Communicating with Vulnerable Patient Populations: A Randomized Intervention to Teach Inpatients to Use the Electronic Patient Portal
}

\author{
Jacob N. Stein ${ }^{1}$ Jared W. Klein ${ }^{1}$ Thomas H. Payne ${ }^{1,2}$ Sara L. Jackson ${ }^{1}$ Sue Peacock ${ }^{1}$
} Natalia V. Oster ${ }^{1}$ Trinell P. Carpenter ${ }^{1}$ Joann G. Elmore ${ }^{3}$

${ }^{1}$ Department of Medicine, University of Washington School of Medicine, Seattle, Washington, United States

2 Information Technology Services, University of Washington School of Medicine, Seattle, Washington, United States

${ }^{3}$ University of California, Los Angeles, California, United States

Appl Clin Inform 2018;9:875-883.
Address for correspondence Joann G. Elmore, MD, MPH, Department of Medicine, UCLA David Geffen School of Medicine, Los Angeles, CA, United States (e-mail: jelmore@mednet.ucla.edu).

\section{Abstract}

Keywords

- patient portal

- discharge summary

- communication barriers

- inpatient care

- discharge planning
Background Patient portals are expanding as a means to engage patients and have evidence for benefit in the outpatient setting. However, few studies have evaluated their use in the inpatient setting, or with vulnerable patient populations.

Objective This article assesses an intervention to teach hospitalized vulnerable patients to access their discharge summaries using electronic patient portals.

Methods Patients at a safety net hospital were randomly assigned to portal use education or usual care. Surveys assessed perceptions of discharge paperwork and the electronic portal.

Results Of the 202 prescreened eligible patients (e.g., deemed mentally competent, spoke English, and had a telephone), only $43 \%$ had working emails. Forty-four percent of participants did not remember receiving or reading discharge paperwork. Patients trained in portal use $(n=47)$ or receiving usual care $(n=23)$ preferred hospitals with online record access ( 85 and $83 \%$, respectively), and felt that online access would increase their trust in doctors ( 85 and $87 \%$ ) and satisfaction with care ( $91 \%$ each). Those who received training in portal use were more likely to register for the portal ( $48 \%$ vs. $11 \% ; p<0.01)$.

Conclusion Patients had positive perceptions of portals, and education increased portal use. Lack of email access is a notable barrier to electronic communication with vulnerable patients.

\section{Background and Significance}

The use of electronic health records (EHRs) throughout the United States health care system has created opportunities for enhancing patient access, engagement, and education. ${ }^{1} \mathrm{~A}$ growing body of evidence suggests that providing patients electronic access to their full medical records may improve their satisfaction, engagement in care, medication adherence, and chronic disease management. ${ }^{2-7}$ Although data suggest

received

June 28, 2018

accepted after revision

October 14, 2018 that black and Hispanic patients are just as interested as Caucasian patients in gaining access to their health information online, they were much less likely to be offered access, raising concerns that the benefits of shared electronic records are not reaching all patients equally. ${ }^{8}$

Most research on patients' access to their EHRs has been conducted in the outpatient ambulatory setting among insured patient populations, although studies are currently underway to improve inpatient portals to engage hospitalized (c) 2018 Georg Thieme Verlag KG Stuttgart · New York
License terms

(1) (1) $\Theta \circledast$ 
patients and vulnerable populations. ${ }^{9-17}$ A 2015 systematic review highlighted the lack of evidence on inpatient portals, as it identified 120 studies on patient portals, but noted that none of the studies included inpatient portals. ${ }^{13}$ Some recent studies have explored the use of bedside portals to allow patients access to their daily laboratory and radiology results with modest benefit, although they did not explore access to discharge summaries or medication lists. ${ }^{16}$ It is important to understand inpatients' access to their EHR information, as hospitalization is a disruptive and consequential event in patients' lives that may signify disease progression, lead to diminished functional capacity, or require new treatments and follow-up. Hospitalization presents an opportunity for education as well as provision of secondary and tertiary prevention of chronic illness, potentially decreasing morbidity and improving quality of life, some of which may be addressed or improved through online record access as seen in results from the outpatient setting.

Unfortunately, after discharge, patients and their families often describe a lack of understanding about prognoses, resources, and posthospitalization care plans, despite providers reporting significant efforts toward education and counseling. ${ }^{18}$ Providing postdischarge instructions for patients has been identified as a potentially important educational strategy to prevent hospital readmission. ${ }^{19,20}$ Providing patients with electronic access to their hospital medical records, including the discharge summary, and access to secure communication with providers may help address this comprehension gap after discharge. ${ }^{21}$ Access to email is often a requirement for portal access and secure messaging, and has thus become a critical piece to enable communication outside of the health care setting.

\section{Objective}

The primary objective of this study was to evaluate an intervention that teaches hospitalized patients at a safety net hospital how to access and use their EHR online portal. Our secondary objectives were to characterize perceptions and access to patient portals, as well as to increase online portal use and uptake by hospitalized patients.

\section{Methods}

\section{Participants and Setting}

Patients were recruited from inpatient internal medicine services at Harborview Medical Center (HMC), a public county hospital operated by the University of Washington School of Medicine. ${ }^{22}$ HMC is an urban safety net hospital with 413 beds and 17,000 annual admissions serving vulnerable patient populations ${ }^{23}$ in King County, Washington, United States. ${ }^{24}$ HMC's diverse patient population includes homeless and incarcerated patients, as well as many patients with complicated or chronic conditions. ${ }^{23}$ Inpatient care at HMC is supported by Cerner Millennium (Kansas City, Missouri, United States) and includes an online portal, "eCare" that can be used to access discharge summaries after hospitalization. Invitation to the patient portal is offered to all patients, via a secure link which is emailed to an email address of their choosing if they opt in.

Patients hospitalized on the internal medicine services during the study period (September 16-October 30, 2016) were prescreened by a physician author (J.S., J.K., or S.J.) using clinical notes to exclude ineligible patients. The prescreening process identified eligible patients who were $\geq 18$ years of age, were able to read and speak English, but did not have diagnoses such as dementia, cognitive impairment, or decompensated psychiatric disorder that would preclude instruction, or discharge to an institutional setting (skilled nursing, long-term care facilities, or incarceration). Given the large number of homeless patients at HMC, "discharge to street" or "discharge to shelter" was considered a destination of "home" and not an institutional setting. The remaining potentially eligible hospitalized patients were then randomized in a 2:1 ratio favoring the intervention. Patients were then personally interviewed by study staff to confirm eligibility including whether or not the patient had access to email and a telephone. Those meeting all eligibility criteria were invited to participate (see - Supplementary Material A, available in the online version). Informed consent was obtained for all participants.

\section{Intervention}

The intervention consisted of an in-person introduction to the online patient portal conducted while participants were still in the hospital. Trained research personnel brought a laptop or tablet to the patient bedside and: (1) determined whether the patient had portal login credentials and sent a new registration email link to patients not yet registered (staff did not assist with actual portal account registration); (2) demonstrated navigation of the patient portal using a simulated teaching (sham) patient account as an example; (3) explained the components of a hospital discharge summary and walked through an example (see - supplementary Material B, available in the online version); and (4) discussed how reading the hospital discharge summary might be helpful to a patient in ways such as reviewing the medication list and sharing the information with a primary care provider or caregiver. Intervention patients were sent two email reminders (not via the portal) by study staff to read their hospital discharge summary after discharge.

Control participants did not receive intervention training or reminder emails, but were invited to use the portal per hospital policy and usual care protocols: nonclinical staff members approach all hospitalized patients shortly after admission as part of the registration process for usual care. Staff members do not use a script and they do not explain details of the patient portal or discharge summaries.

\section{Measures and Data Collection}

Patient demographics (e.g., age, sex, race) and comorbidities (e.g., coronary artery disease, diabetes, hypertension, cancer, human immunodeficiency virus, substance use disorder) were abstracted from the EHR by a study physician (J.S., J. K., or S.J.).

The baseline survey was conducted in person during the hospitalization. The survey assessed patients' understanding 
of their medications and medical problems, a previously validated single-item health literacy assessment about their confidence in filling out forms without help ${ }^{22,25}$ and perceived helpfulness of reading the discharge summary, using a 4-point Likert scale.

The follow-up survey was administered beginning 2 weeks after hospital discharge. Patients were sent one email invitation and up to three telephone call reminders to complete the postdischarge survey. Patients with a nonworking phone number were sent an additional email from study staff and those with a nonworking email received an additional phone call. Paper questionnaires were mailed to patients who could not be reached via email or telephone. Thus, depending on which reminder patients responded to, the postdischarge survey was completed either via the Web, telephone, or paper. The postdischarge survey included the same questions as the baseline survey, but also assessed patients' perceptions and self-reported utilization of the patient portal after discharge, their trust in their doctors, and satisfaction with their care. Upon completing the postdischarge survey, participants were given a $\$ 25$ gift card for participating in the study.

All survey data were managed using the Research Electronic Data Capture Web application (Vanderbilt, Nashville, Tennessee, United States), including data originally completed via telephone or mailed paper survey ${ }^{26}$ (see -Supplementary Material C for the complete questionnaires, available in the online version).

\section{Statistical Analysis}

Frequency distributions of demographics, survey, and hospital log data were analyzed across intervention and control groups. Comparisons were made using Pearson's chi-square test or Fisher's exact test, where appropriate. Four-point Likert scale items were dichotomized for analytical purposes. The primary outcome was whether they accessed the patient portal, as determined by electronic logs that tracked which patients created a patient portal account. We also tracked whether patients had a working email address as this was a primary barrier to study participation. All analyses were conducted using SAS 9.4 (SAS, Cary, North Carolina, United States).

\section{Results}

Of 533 hospitalized patients on the internal medicine services during the study period, 192 were deemed ineligible from the prescreening chart review, 85 were not available for approach by research staff during their hospitalization because they were undergoing medical testing or procedures, and 27 patients declined to participate before eligibility could be determined. Of the remaining 229 patients, 114 (50\%) were deemed ineligible during the in-person screening (see the figure in - Supplementary Material A, available in the online version), primarily due to lack of an email address and 8 declined participation. An additional 21 patients who did participate in the study did not have working email addresses. Thus, among the 202 hospitalized patients who were deemed mentally competent, spoke English, and had a telephone, only 86 (43\%) had a working email (-Fig. 1).

Seventy patients (47 intervention and 23 control) completed all components of the study including the postdischarge survey. For patients randomized to the intervention, the educational component took an average of approximately 15 minutes to complete (range: 5-30 minutes).

The study population is described in -Table 1. The majority of patients were 50 years or older, male, white, and non-Hispanic. Males represented a greater proportion of the control group than the intervention group $(p=0.03)$. At baseline, while most patients reported understanding their

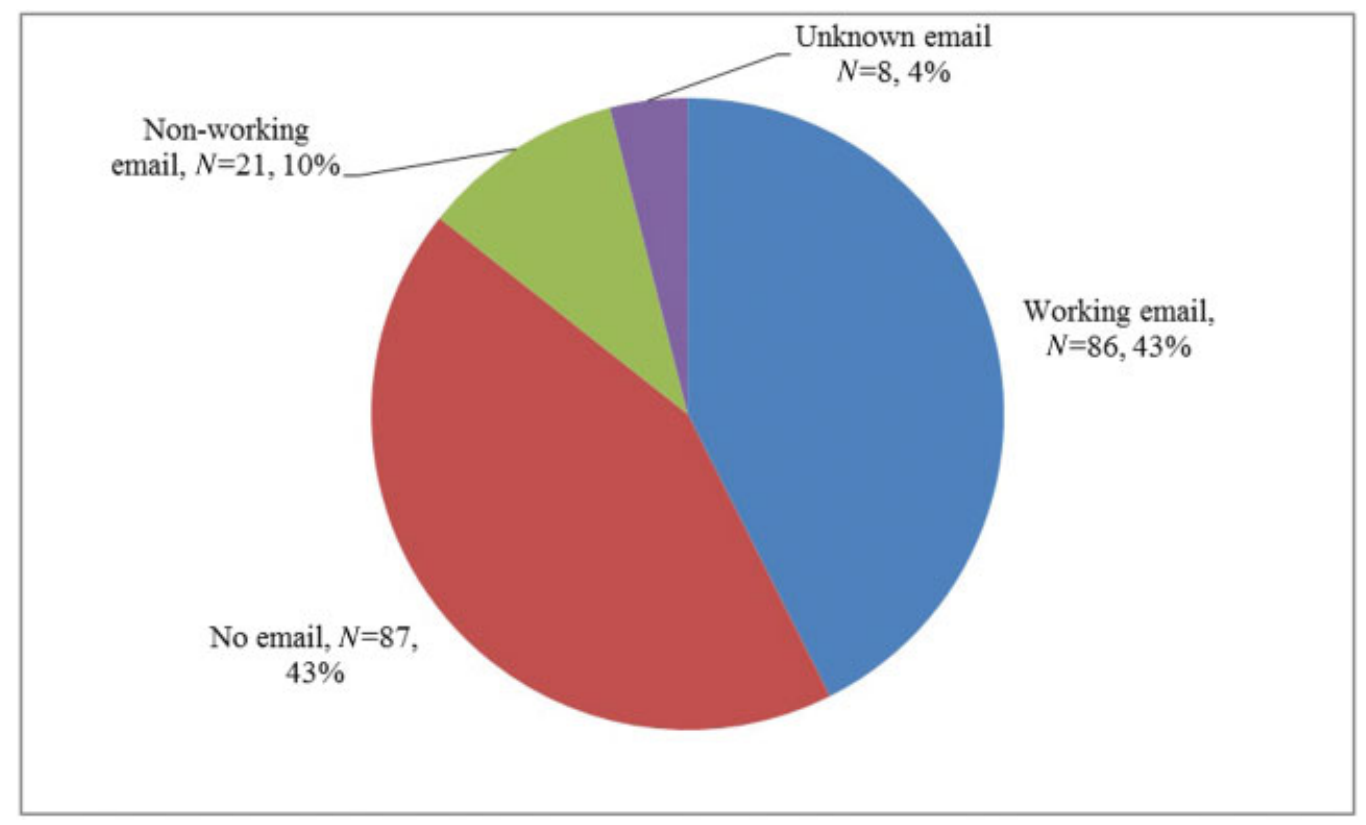

Fig. 1 Email use among 202 vulnerable safety net hospital patients who were English speaking, did not have a diagnosis of a condition that would affect cognition, and were discharged to home. 
Table 1 Patient characteristics and baseline health knowledge

\begin{tabular}{|c|c|c|c|}
\hline \multirow[t]{3}{*}{ Patient characteristics } & \multicolumn{2}{|l|}{ Study arm } & \multirow[t]{3}{*}{$p$-Value } \\
\hline & Intervention, $N(\%)$ & Control, $N(\%)$ & \\
\hline & $47(67)$ & $23(33)$ & \\
\hline \multicolumn{4}{|l|}{ Sex } \\
\hline Female & $21(45)$ & $4(17)$ & \multirow[t]{2}{*}{0.025} \\
\hline Male & $26(55)$ & $19(83)$ & \\
\hline \multicolumn{4}{|l|}{ Race } \\
\hline White & $34(72)$ & $19(83)$ & \multirow[t]{4}{*}{0.88} \\
\hline Black & $5(11)$ & $2(9)$ & \\
\hline Asian & $3(6)$ & $1(4)$ & \\
\hline Other & $5(11)$ & $1(4)$ & \\
\hline \multicolumn{4}{|l|}{ Ethnicity } \\
\hline Hispanic & $5(11)$ & $3(13)$ & \multirow[t]{2}{*}{$>0.99$} \\
\hline Not Hispanic & $42(89)$ & $20(87)$ & \\
\hline \multicolumn{4}{|l|}{ Number of comorbidities ${ }^{a}$} \\
\hline $0-1$ & $10(21)$ & $7(30)$ & \multirow[t]{3}{*}{0.70} \\
\hline $2-3$ & $17(36)$ & $7(30)$ & \\
\hline $4+$ & $20(43)$ & $9(39)$ & \\
\hline \multicolumn{4}{|l|}{ Most common comorbidities } \\
\hline Substance abuse & $21(45)$ & $7(30)$ & 0.25 \\
\hline Hypertension & $15(32)$ & $10(43)$ & 0.34 \\
\hline Diabetes & $13(28)$ & $5(22)$ & 0.59 \\
\hline Pain & $9(19)$ & $5(22)$ & $>0.99$ \\
\hline Depression & $8(17)$ & $6(26)$ & 0.53 \\
\hline \multicolumn{4}{|l|}{ Age } \\
\hline $18-49$ & $18(38)$ & $9(39)$ & \multirow[t]{2}{*}{0.95} \\
\hline $50+$ & $29(62)$ & $14(61)$ & \\
\hline \multicolumn{4}{|l|}{ Baseline survey knowledge $^{\mathrm{b}}$} \\
\hline \multicolumn{4}{|l|}{ Know what medications to take } \\
\hline Disagree/Strongly disagree & $11(23)$ & $2(9)$ & \multirow[t]{2}{*}{0.20} \\
\hline Agree/Strongly agree & $36(77)$ & $21(91)$ & \\
\hline \multicolumn{4}{|l|}{ Know what medications do } \\
\hline Disagree/Strongly disagree & $8(17)$ & $3(13)$ & \multirow[t]{2}{*}{$>0.99$} \\
\hline Agree/Strongly agree & $39(83)$ & $20(87)$ & \\
\hline \multicolumn{4}{|l|}{ Know when to visit physician } \\
\hline Disagree/Strongly disagree & $10(21)$ & $2(9)$ & \multirow[t]{2}{*}{0.31} \\
\hline Agree/Strongly agree & $37(79)$ & $21(91)$ & \\
\hline \multicolumn{4}{|c|}{ Understand why admitted to hospital } \\
\hline Disagree/Strongly disagree & $2(4)$ & $0(0)$ & \multirow[t]{2}{*}{$>0.99$} \\
\hline Agree/Strongly agree & $45(96)$ & $23(100)$ & \\
\hline \multicolumn{4}{|c|}{ Understand what need to do after leaving hospital } \\
\hline Disagree/Strongly disagree & $5(11)$ & $4(17)$ & \multirow[t]{2}{*}{0.46} \\
\hline Agree/Strongly agree & $42(89)$ & $19(83)$ & \\
\hline
\end{tabular}




\begin{tabular}{|c|c|c|c|}
\hline \multirow[t]{3}{*}{ Patient characteristics } & \multicolumn{2}{|l|}{ Study arm } & \multirow[t]{3}{*}{$p$-Value } \\
\hline & Intervention, $N(\%)$ & Control, $N(\%)$ & \\
\hline & $47(67)$ & $23(33)$ & \\
\hline \multicolumn{4}{|c|}{ Good idea if patients could read hospital records } \\
\hline Disagree/Strongly disagree & $3(6)$ & $1(4)$ & \multirow[t]{2}{*}{$>0.99$} \\
\hline Agree/Strongly agree & $44(94)$ & $22(96)$ & \\
\hline \multicolumn{4}{|l|}{ Confidence in filling out forms } \\
\hline Somewhat/A little bit/Not at all & $11(23)$ & $6(26)$ & \multirow[t]{2}{*}{0.81} \\
\hline Extremely/Quite a bit & $36(77)$ & $17(74)$ & \\
\hline
\end{tabular}

Abbreviation: HIV, human immunodeficiency virus.

${ }^{a}$ Comorbidities captured include: pain, diabetes, hypertension, coronary artery disease, chronic obstructive pulmonary disease, chronic kidney disease, heart failure, atrial fibrillation, substance abuse disorder, depression, anxiety, cirrhosis, stroke, cancer, end-stage renal disease, deep vein thrombosis/pulmonary embolism, hepatitis C, obstructive sleep apnea, HIV, and other.

baseline survey results.

medications, why they were admitted to the hospital, and what to do after they left the hospital, it is also relevant to note that $19 \%$ of combined samples reported not knowing what medications to take and 11 to $17 \%$ did not know what they needed to do after leaving the hospital. The majority of patients also reported confidence filling out forms themselves, and thought it was a good idea if patients had access to their own hospital records. There were no differences in responses to these questions between the intervention and control groups.

-Table 2 summarizes the data collected after hospital discharge. The majority of the intervention patients (89\%) felt the training they received to access the patient portal was useful. Both the intervention and the control patients overwhelmingly preferred hospitals with online record access ( 85 and $83 \%$, respectively), feeling that access to medical records would increase their trust in doctors ( 85 and $87 \%$, respectively) and their satisfaction with care (91\% for each group). Patients in both the study groups were interested in the idea of sharing their hospitalization medical records with others, particularly with their primary care doctor and family member.

Over half of the patients in both the intervention and control study arms reported wishing they had more information about their recent hospitalization (57 and 67\%, respectively). Patients were also asked in the postdischarge survey about the hard copy paperwork all patients are given upon discharge from the hospital ( - Fig. 2). As the results were similar between patients in both the study arms, the data are merged for clarity. A substantial proportion did not remember receiving the papers or did not read them (44\%), did not find them helpful (37\%), and did not know where the paperwork was at the time of the postdischarge survey (20\%).

Based on electronic logs, patients who received the intervention training in accessing and navigating the patient portal were significantly more likely to register for the patient portal ( $48 \%$ vs. $11 \%, p<0.01)$. According to patient self-report, intervention patients were also more likely to attempt logging into the portal; this reached borderline statistical significance ( $60 \%$ vs. $35 \%, p=0.05$ ). The more frequent reasons given for not accessing the portal by our patients were personal/time constraints, or not thinking about accessing it, rather than technical difficulties.

\section{Discussion}

This study suggests that vulnerable patient populations are enthusiastic about electronic patient portals, yet a surprising percentage do not use email. Email is the primary method of communication used by most health systems for both signing patients up for portals and encouraging them to read their doctors' notes, and yet only $43 \%$ of vulnerable inpatients in this study had a working email address. ${ }^{27}$ This is an important and unexpected finding that almost half of patients approached for this study did not have working email. During prescreening, we had excluded patients with cognitive or language barriers, and thus had expected a much higher percentage of remaining patients to report using email. We consider the inability to reach patients by email to be critical as many patient portals used by hospitals and clinics nationwide require email for account access and to prompt patients to read clinic notes. ${ }^{27}$ When developing an educational program for patients surrounding portal use, including information on how to access and use email, offering other methods to access the portal may be needed, especially if we hope to reach vulnerable patients. ${ }^{27}$

In this study, we also found that patients are more likely to take steps to register for the patient portal if they are provided with brief training. Most patients indicated a preference for hospitals that offer access to patient portals and also reported that they would have increased satisfaction with their health care and trust in their physicians if they had online access to their hospital records, both relevant findings in an era of patient satisfaction scores. Given the high percentage of patients who either did not read (44\%) or could not find (20\%) the hard copy paperwork given to them at discharge, online portals may improve patient engagement by allowing discharged patients to read the hospital 
Table 2 Posthospital discharge follow-up data on perception and activity related to electronic patient portals

\begin{tabular}{|c|c|c|c|}
\hline & \multicolumn{2}{|l|}{ Study arm } & \multirow[t]{3}{*}{$p$-Value ${ }^{b}$} \\
\hline & Intervention, $N(\%)$ & Control, $N(\%)$ & \\
\hline & $47(67)$ & $23(33)$ & \\
\hline \multicolumn{4}{|l|}{ Perception } \\
\hline \multicolumn{4}{|c|}{ Training I received about the patient portal was useful } \\
\hline Disagree/Strongly disagree & $5(11)$ & NA & \\
\hline Agree/Strongly agree & $41(89)$ & NA & \\
\hline \multicolumn{4}{|c|}{ Wish had more information about hospitalization } \\
\hline Disagree/Strongly disagree & $20(43)$ & $7(33)$ & \multirow[t]{2}{*}{0.47} \\
\hline Agree/Strongly agree & $27(57)$ & $14(67)$ & \\
\hline \multicolumn{4}{|l|}{ Prefer hospital with online record access } \\
\hline No & $2(4)$ & $1(4)$ & \multirow[t]{3}{*}{$>0.99$} \\
\hline Yes & $40(85)$ & $19(83)$ & \\
\hline Don't know & $5(11)$ & $3(13)$ & \\
\hline \multicolumn{4}{|c|}{ Access to medical records would increase my trust in doctors } \\
\hline Disagree/Strongly disagree & $7(15)$ & $3(13)$ & \multirow[t]{2}{*}{$>0.99$} \\
\hline Agree/Strongly agree & $40(85)$ & $20(87)$ & \\
\hline \multicolumn{4}{|c|}{ Access to medical records would increase my satisfaction with care } \\
\hline Disagree/Strongly disagree & $4(9)$ & $2(9)$ & \multirow[t]{2}{*}{$>0.99$} \\
\hline Agree/Strongly agree & $43(91)$ & $21(91)$ & \\
\hline \multicolumn{4}{|l|}{ Would share medical records with ${ }^{\mathrm{a}}$} \\
\hline Share records with family & $25(53)$ & $13(57)$ & 0.79 \\
\hline Share records with primary care doctor & $37(79)$ & $22(96)$ & 0.087 \\
\hline Share records with caregiver & $16(34)$ & $9(39)$ & 0.68 \\
\hline No one & $8(17)$ & $0(0)$ & 0.046 \\
\hline \multicolumn{4}{|l|}{ Activity postdischarge } \\
\hline \multicolumn{4}{|l|}{ Patient activity on portal (hospital click data) ${ }^{c}$} \\
\hline Declined portal registration & $6(13)$ & $8(42)$ & \multirow[t]{3}{*}{0.005} \\
\hline Did not register & $18(39)$ & $9(47)$ & \\
\hline Registered & $22(48)$ & $2(11)$ & \\
\hline \multicolumn{4}{|l|}{ Attempted to login to patient portal } \\
\hline No & $19(40)$ & $15(65)$ & \multirow[t]{2}{*}{0.051} \\
\hline Yes & $28(60)$ & $8(35)$ & \\
\hline \multicolumn{4}{|l|}{ Reasons given for not logging into the portal ${ }^{\mathrm{a}}$} \\
\hline Didn't know about it & $2(4)$ & $5(22)$ & 0.035 \\
\hline Technical difficulties & $5(11)$ & $3(13)$ & $>0.99$ \\
\hline Didn't feel well enough & $6(13)$ & $2(9)$ & $>0.99$ \\
\hline Personal or time constraints & $11(23)$ & $5(22)$ & 0.88 \\
\hline Didn't think about it & $10(21)$ & $6(26)$ & 0.65 \\
\hline Forgot password & $3(6)$ & $3(13)$ & 0.39 \\
\hline Other reason & $12(26)$ & $6(26)$ & 0.96 \\
\hline
\end{tabular}

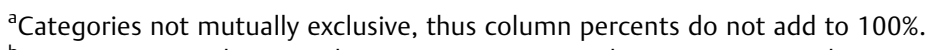

${ }^{\mathrm{b}}$ Comparisons made using chi-square statistic or Fisher's exact tests where appropriate.

'Patients who declined portal registration indicated that they did not want to receive email information instructing them how to sign up for the portal. Patients who did not register received the email information describing how to register but did not complete the registration process online. 


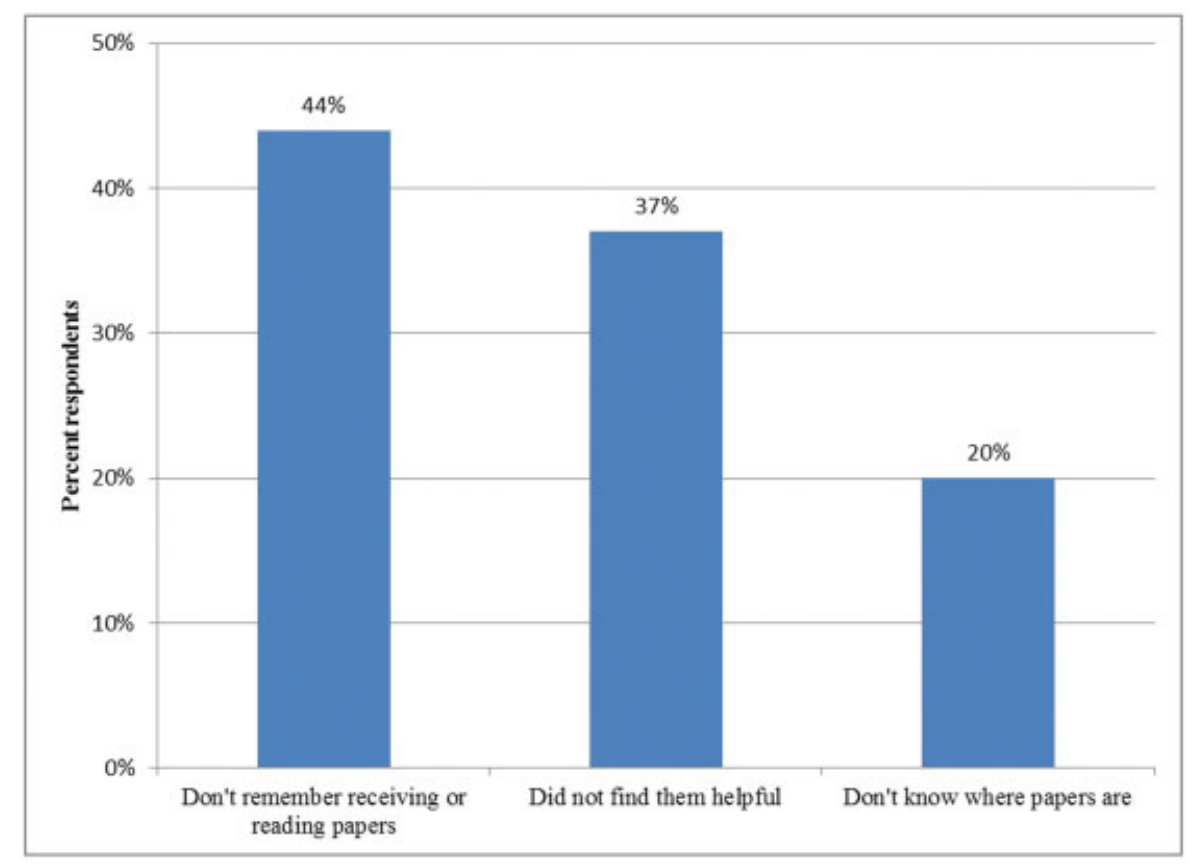

Fig. 2 Patients' responses to a survey question about hospital discharge paperwork. Results for patients from the intervention and control arms were similar and are therefore combined.

paperwork at their convenience a long time after returning home, while at the same time providing a secure, easy-tofind location for accessing their records. Our training likely improved awareness of the resource, and may have enhanced patient activation and comfort with the online tool, all of which may have contributed to the observed increase in use. Training patients to access their health information using portals on busy inpatient wards, without the benefit of study staff, is a challenge. However, this work could be incorporated into transitions of care processes developed to improve care quality and continuity of information between in- and outpatient domains.

Our findings among this vulnerable patient population are consistent with prior research demonstrating that most patients consider accessing their health information via online portals to be important. ${ }^{28,29}$ Unfortunately, many patients, especially those from vulnerable populations, do not use patient portals either because they are not given the opportunity ${ }^{28}$ or because they do not have the ability to access them. ${ }^{30-32}$ Recent studies are consistent with our findings and suggest that tailored and accessible training and support to assist such patient populations with portal use was strongly needed, and beneficial when provided. ${ }^{12-14,33,34}$ U.S. Census data from 2015 shows that the majority of Asian and nonHispanic white households reported Internet use at home ( 88 and $79 \%$, respectively), compared with lower rates in Hispanic and black households (70 and 64\%, respectively). ${ }^{35} \mathrm{~A}$ Pew Research Center report on U.S. smartphone use in 2015 states that $13 \%$ of Hispanic and $12 \%$ of black smartphone users are "smartphone dependent" for Internet access due to lack of broadband Internet at home. The report also notes that $73 \%$ of Hispanic and $67 \%$ of black smartphone owners in the United States used their phones to seek information about a health condition in the prior year, compared with $58 \%$ of white smartphone owners. ${ }^{36}$ Among vulnerable populations in particular, finding methods to communicate is challenging and, as our results suggest, may require the identification and implementation of novel strategies that do not involve email.

Our study has several limitations. It was notable for the high rate of exclusion due to diagnoses of dementia, non-English fluency, or lack of email access. The final small sample size might have limited the randomization, with more men in the control group, and limited our ability to detect meaningful differences between the randomized groups. The study was conducted at a single safety net hospital that serves a vulnerable patient population, thereby potentially limiting the external validity of our findings. However, it is plausible that patients in other large urban areas would experience similar benefits and challenges. Portal access was measured by registration for an account as a surrogate, rather than by tracking who actually opened their discharge summary, as it was not possible to extract that level of individualized click data. Lastly, the survey data relied on self-report.

Strengths of this study include that it was conducted in an inpatient setting serving vulnerable patients and that it assessed the patient perspective on current methods of communication being used by hospitals (e.g., discharge papers and email). We demonstrated that increasing access to the patient portal is possible in an urban safety net hospital and patients were enthusiastic about this new tool, yet lack of email is a critical issue that needs to be addressed.

Future interventions could emphasize signing patients up for email accounts or could explore alternative ways to share health information with patients, such as texting or app-based interfaces. Secure hospital- or clinic-based kiosks could offer a safe means to access portals after discharge. Access could also be offered to caregivers or surrogates of patients with dementia or other functional impairments. Future studies could also 
explore clinical endpoints to bolster our understanding of the effects of shared medical records on patient engagement, understanding, satisfaction, and health outcomes.

\section{Conclusion}

Hospitals need to support the transition of care from the hospital back to the community and this can include education and providing patients access to patient portals and their electronic discharge summaries. Vulnerable patients show interest in having this information available to them and efforts should be made to engage them and not allow the digital divide to grow. As shown by our study in a safety net hospital, additional efforts to teach patients how to access the portal may increase patient engagement. However, as email may not be an ideal method for communication with all patients, hospitals should consider other means of communication or consider adding the support of patient navigators.

\section{Clinical Relevance Statement}

Electronic health records are widely used, and patient portals are rapidly growing as a means to engage patients in their care outside of a health care setting. We developed a brief intervention to increase uptake of patient portals, and demonstrated efficacy in an urban safety net hospital. However, many underserved patients lacked email access, highlighting a critical barrier to connect with vulnerable patients via portals.

\section{Multiple Choice Questions}

1. Which of the following statements best describe the attitudes of participants in this study in regards to online medical record access?

a. Increased trust in physicians.

b. Increased satisfaction with care.

c. Prefer a hospital which offers online record access.

d. All of the above.

Correct Answer: The correct answer is option d. While participants attitudes were similar in both study arms, as seen in - Table 2, patients endorsed that online record access would increase trust in physicians (87\% control, $85 \%$ intervention), increase satisfaction in care ( $91 \%$ both groups), and voiced a preference for hospitals that offered online medical record access (83\% control, $85 \%$ intervention). These results are similar to findings in studies conducted in the outpatient settings that indicate robust interest and support for patient portals and online record access.

2. Which of the following most accurately summarizes the findings of the study regarding communication with patients after a hospital stay?

a. Email is a widely available method of communication with near universal uptake, and is ideal for patient outreach.

b. The vast majority of patients prefer their communication via hard copy printed instructions. c. Creative approaches are needed, as email use is not as prevalent as expected, and hard copy paperwork is often misplaced.

d. Most patients are not particularly interested in communication or information after hospital discharge.

Correct Answer: The correct answer is option c. As seen in - Fig. 1, only $43 \%$ of patients had a functional email address, a surprisingly low figure that suggests email does not have as wide uptake as expected, and is a limited method for communication with patients. -Fig. 2 describes attitudes toward hard copy paperwork, and a high proportion (44\%) did not recall receiving them or reading them, did not find them helpful (37\%), or no longer knows where those papers are (20\%), suggesting that this is not a preferred form of communication. Lastly, in -Table 2, over half of patients in both groups desired more information about their hospitalization (57 and 67\%, respectively), and had overwhelming preference for access to medical records. Thus, as mentioned in the "Discussion" section, answer choice $C$ best describes the complexity and challenges in communicating with patients, and the need for creative solutions going forward, such as texting or app-based interfaces.

\section{Protection of Human and Animal Subjects}

This study was performed in compliance with the World Medical Association Declaration of Helsinki on Ethical Principles for Medical Research involving Human Subjects and was reviewed by the University of Washington Institutional Review Board.

\section{Funding}

This study was generously funded by the Robert Wood Johnson Foundation, the Gordon \& Betty Moore Foundation, the Peterson Center on Healthcare, and the Cambia Health Foundation.

\section{Conflict of Interest}

None declared.

\section{Acknowledgments}

We acknowledge the patients at Harborview Medical Center, first and foremost, for inspiring us to improve our care, and for participating in the study. We also acknowledge the assistance of the Institute of Translational Health Sciences (ITHS) in data collection.

\section{References}

1 Walker J, Meltsner M, Delbanco T. US experience with doctors and patients sharing clinical notes. BMJ 2015;350:g7785

2 Bronson DL, Costanza MC, Tufo HM. Using medical records for older patient education in ambulatory practice. Med Care 1986; 24(04):332-339

3 Ross SE, Moore LA, Earnest MA, Wittevrongel L, Lin CT. Providing a web-based online medical record with electronic communication capabilities to patients with congestive heart failure: randomized trial. J Med Internet Res 2004;6(02):e12 
4 Pagliari C, Detmer D, Singleton P. Potential of electronic personal health records. BMJ 2007;335(7615):330-333

5 Wright E, Darer J, Tang X, et al. Sharing physician notes through an electronic portal is associated with improved medication adherence: quasi-experimental study. J Med Internet Res 2015;17(10): e226

6 Esch T, Mejilla R, Anselmo M, Podtschaske B, Delbanco T, Walker J. Engaging patients through open notes: an evaluation using mixed methods. BMJ Open 2016;6(01):e010034

7 Delbanco T, Walker J, Bell SK, et al. Inviting patients to read their doctors' notes: a quasi-experimental study and a look ahead. Ann Intern Med 2012;157(07):461-470

8 Dhanireddy S, Walker J, Reisch L, Oster N, Delbanco T, Elmore JG. The urban underserved: attitudes towards gaining full access to electronic medical records. Health Expect 2014;17(05):724-732

9 Masterson Creber R, Prey J, Ryan B, et al. Engaging hospitalized patients in clinical care: Study protocol for a pragmatic randomized controlled trial. Contemp Clin Trials 2016;47:165-171

10 Woollen J, Prey J, Wilcox L, et al. Patient experiences using an inpatient personal health record. Appl Clin Inform 2016;7(02): 446-460

11 Delbanco T, Walker J, Darer JD, et al. Open notes: doctors and patients signing on. Ann Intern Med 2010;153(02):121-125

12 Huerta TR, McAlearney AS, Rizer MK. Introducing a patient portal and electronic tablets to inpatient care. Ann Intern Med 2017;167 (11):816-817

13 Irizarry T, DeVito Dabbs A, Curran CR. Patient portals and patient engagement: a state of the science review. J Med Internet Res 2015;17(06):e148

14 Prey JE, Woollen J, Wilcox L, et al. Patient engagement in the inpatient setting: a systematic review. J Am Med Inform Assoc 2014;21(04):742-750

15 Hefner JL, Sieck CJ, McAlearney AS. Training to optimize collaborative use of an inpatient portal. Appl Clin Inform 2018;9(03): 558-564

16 Walker DM, Menser T, Yen PY, McAlearney AS. Optimizing the user experience: identifying opportunities to improve use of an inpatient portal. Appl Clin Inform 2018;9(01):105-113

17 Griffin A, Skinner A, Thornhill J, Weinberger M. Patient portals: who uses them? What features do they use? And do they reduce hospital readmissions? Appl Clin Inform 2016;7(02):489-501

18 Paterson B, Kieloch B, Gmiterek J. 'They never told us anything': postdischarge instruction for families of persons with brain injuries. Rehabil Nurs 2001;26(02):48-53

19 Herzig SJ, Schnipper JL, Doctoroff L, et al. Physician perspectives on factors contributing to readmissions and potential prevention strategies: a multicenter survey. J Gen Intern Med 2016;31(11): 1287-1293

20 Scotten M, Manos EL, Malicoat A, Paolo AM. Minding the gap: interprofessional communication during inpatient and post discharge chasm care. Patient Educ Couns 2015;98(07):895-900

21 Feldman HJ, Walker J, Li J, Delbanco T. OpenNotes: hospitalists' challenge and opportunity. J Hosp Med 2013;8(07):414-417
22 Sheffield JV, Young A, Goldstein EA, Logerfo JP. The public hospital mission at Seattle's Harborview Medical Center: high-quality care for the underserved and excellence in medical education. Acad Med 2006;81(10):886-890

23 UW Medicine Harborview Medical Center. Available at: http://www. uwmedicine.org/about/Documents/HMC-FS-final.pdf. Accessed June 11,2017

24 U.S. Census Bureau Population Division. Annual Estimates of the Resident Population: April 1, 2010 to July 1, 2016. 2017. Available at: https://factfinder.census.gov/faces/nav/jsf/pages/index.xhtml. Accessed October, 2018

25 Chew LD, Griffin JM, Partin MR, et al. Validation of screening questions for limited health literacy in a large VA outpatient population. J Gen Intern Med 2008;23(05):561-566

26 Harris PA, Taylor R, Thielke R, Payne J, Gonzalez N, Conde JG. Research electronic data capture (REDCap)-a metadata-driven methodology and workflow process for providing translational research informatics support. J Biomed Inform 2009;42(02):377-381

27 Mafi JN, Mejilla R, Feldman H, et al. Patients learning to read their doctors' notes: the importance of reminders. J Am Med Inform Assoc 2016;23(05):951-955

28 Peacock S, Reddy A, Leveille SG, et al. Patient portals and personal health information online: perception, access, and use by US adults. J Am Med Inform Assoc 2017;24(e1):e173-e177

29 Wen KY, Kreps G, Zhu F, Miller S. Consumers' perceptions about and use of the internet for personal health records and health information exchange: analysis of the 2007 Health Information National Trends Survey. J Med Internet Res 2010;12(04):e73

30 McCloud RF, Okechukwu CA, Sorensen G, Viswanath K. Beyond access: barriers to internet health information seeking among the urban poor. J Am Med Inform Assoc 2016;23(06):1053-1059

31 Hargittai E. Second-level digital divide: differences in people's online skills. First Monday 2002;7(04). Available at: http://webuse. org/pdf/Hargittai-SecondLevelFM02.pdf. Accessed December 6, 2018

32 Jawhari B, Keenan L, Zakus D, et al. Barriers and facilitators to electronic medical record (EMR) use in an urban slum. Int J Med Inform 2016;94(Oct):246-254

33 Tieu L, Schillinger D, Sarkar U, et al. Online patient websites for electronic health record access among vulnerable populations: portals to nowhere? JAMA 2016

34 Schickedanz A, Huang D, Lopez A, et al. Access, interest, and attitudes toward electronic communication for health care among patients in the medical safety net. J Gen Intern Med 2013;28(07):914-920

35 Bureau USCComputer and Internet Use in the United States; 2015. Available at: https://www.census.gov/content/dam/Census/library /publications/2017/acs/acs-37.pdf. Accessed June 2018

36 Anderson M. Racial and ethnic differences in how people use mobile technology. FactTank: News in the Numbers; 2015. Available at: http://www.pewresearch.org/fact-tank/2015/04/30/racial-and-eth nic-differences-in-how-people-use-mobile-technology/. Accessed February 15, 2017 